$11^{\text {th }}$ International Semiconducting and Insulating Materials Conference held at Canberra, Australia, 3-7 July, 2000.

\title{
Selectively Excited Blue Luminescence in heavily Mg doped p-type GaN
}

\author{
John S. Colton and Peter Y. Yu \\ Department of Physics, University of California, Berkeley, CA 94720, USA \\ and \\ Materials Sciences Division, Lawrence Berkeley National Laboratory, \\ Berkeley, CA 94720 USA
}

\begin{abstract}
The emission at $\sim 2.8 \mathrm{eV}$ from heavily doped p-GaN, known as the blue luminescence (BL), has been studied by selective excitation using a dye laser tunable between 2.7-3.0 eV. The peak position and intensity of the BL are found to exhibit an unusual dependence on the excitation photon energy. We have explained our results with a shallow-donor and deep-acceptors pair recombination model which includes potential fluctuations induced by heavy doping. We found a "critical energy" of $\sim 2.8 \mathrm{eV}$ for the BL. Electron-hole pairs with energies above this energy are able to achieve quasithermal equilibrium while those with energies below $2.8 \mathrm{eV}$ are strongly "localized".
\end{abstract}

It is now well known that heavily doped p-type $\mathrm{GaN}$ often exhibits a strong broadband luminescence with a peak centered around 2.8-2.9 eV in the blue[1]. This blue luminescence (BL) peak behaves differently than the higher energy emission around 3.1-3.2 eV which has been attributed to recombination at shallow impurity centers. There is no consensus in the identification of the BL. Most authors agree that the BL originates from donor-acceptor pair (DAP) recombination, however, they disagree on the nature of the donors and acceptors. For example, optically detector magnetic resonance (ODMR) study in a Mg-doped bulk sample has led to the suggestion that complexes containing deep donors are involved[2]. On the other hand, similar ODMR studies in thin film samples have led to the conclusion that the BL originates from deep acceptors and shallow donors [3]. Recently, Suski [4] has measured the pressure dependence of the BL in bulk GaN samples and concluded that deep donors are involved. In this paper we report an investigation of the BL in a heavily doped $\mathrm{GaN}: \mathrm{Mg}$ thin film sample grown by metal-organic chemical vapor deposition (MOCVD) on sapphire by selective excitation with a tunable dye laser. This technique has also been applied to study the yellow luminescence (YL) in n-type GaN[5]. Unlike the results in YL, the peak energy of the resonantly excited BL varies in an unusual way with the excitation photon energy. Our results have been explained by assuming that the BL contains at least two components. Those excited by photon energy $(\hbar \omega)$ less than $\sim 2.8 \mathrm{eV}$ originate from strongly localized centers while those with $\hbar \omega>2.8 \mathrm{eV}$ come from interacting centers so that their carriers can achieve quasi-thermal equilibrium.

Our GaN:Mg sample is a 2 microns thick film grown on sapphire by MOCVD. It was doped with around $10^{19} \mathrm{~cm}^{-3}$ of $\mathrm{Mg}$ atoms during growth, followed by annealing at $750^{\circ} \mathrm{C}$ for 4 minutes. The measured carrier concentration is $\sim 2 \times 10^{17} \mathrm{~cm}^{-3}$. These values are typical of $\mathrm{GaN}$ samples which have been reported to display the BL in the literature[6].

The photoluminescence (PL) of our sample at temperature $\mathrm{T}=12 \mathrm{~K}$ when excited by a $\mathrm{HeCd}$ laser at $325 \mathrm{~nm}$ exhibits a $\sim 0.3 \mathrm{eV}$ wide BL peak centered around $2.8 \mathrm{eV}$. Since it resembles the BL reported by other authors[1], it will not be reproduced here. A Spectra Physics UV $\mathrm{Ar}^{+}$-ion laser pumped dye laser (dye: Stilbene 420) with a tuning range of 2.67 - 
$11^{\text {th }}$ International Semiconducting and Insulating Materials Conference held at Canberra, Australia, 3-7 July, 2000.

$3.00 \mathrm{eV}$ was used to selectively excite the BL which was analyzed by a Spex double spectrometer and detected by photon counting. The selectively excited PL spectra, in a semi$\log$ plot, are shown in Fig. 1 as a function of the difference between the emission photon energy $\left(\hbar \omega_{\mathrm{e}}\right)$ and the excitation laser energy $\left(\hbar \omega_{\mathrm{i}}\right)$. The small oscillations that modulate the PL results from Fabry-Perot interference between light reflected from the two surfaces of the film.

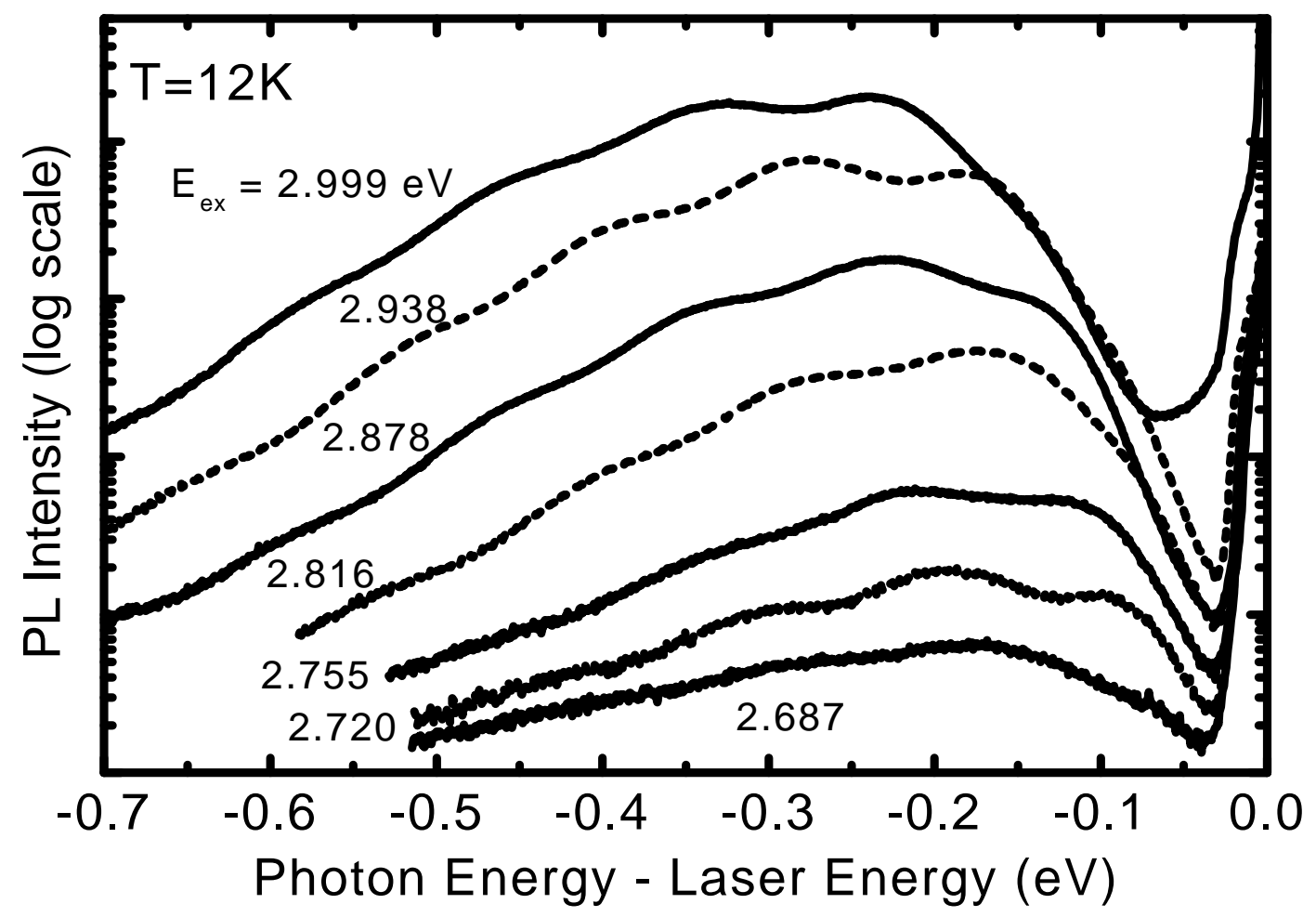

Fig 1. Low temperature PL of the thin film GaN:Mg sample selectively excited by dye laser at photon energies between $2.687 \mathrm{eV}-2.999 \mathrm{eV}$. Notice how the PL intensity increases exponentially with the excitation photon energy.

Optically excited emission can usually be classified either as PL or Raman scattering (RS) depending on the way $\hbar \omega_{\mathrm{e}}$ changes with $\hbar \omega_{\mathrm{i}}$. In case of $\mathrm{RS}, \hbar \omega_{\mathrm{e}}$ follows $\hbar \omega_{\mathrm{i}}$ at a constant energy separation $\Delta \mathrm{E}=\left(\hbar \omega_{\mathrm{e}}-\hbar \omega_{\mathrm{i}}\right)$ with $\omega_{\mathrm{i}}-\omega_{\mathrm{e}}$ defined as the Raman frequency. On the other hand, $\hbar \omega_{\mathrm{e}}$ of PL is constant and independent of $\hbar \omega_{1}$. Hence a plot of $\Delta \mathrm{E}$ as a function of $\hbar \omega_{i}$ for PL would be a straight line with a slope of -1 . In case of the BL we found a rather unusual behavior. For $\hbar \omega_{1}<2.8 \mathrm{eV}, \Delta \mathrm{E}$ is constant as in RS and the Raman frequency is equal to $\sim 180$ meV. However, for $\hbar \omega_{i}>2.8 \mathrm{eV}, \Delta \mathrm{E}$ starts to change with $\hbar \omega_{i}$ until it becomes a straight line as in PL but with a slope of $\sim-0.43$ ! These results are shown in Figure 2.

Another interesting feature of the BL curves in Fig. 1 is the exponential increase in the integrated BL intensity with $\hbar \omega_{1}$ as $\sim \exp \left(\hbar \omega_{1} / \mathrm{E}_{0}\right)$ with $\mathrm{E}_{0}=33 \mathrm{meV}$. We have also observed thermal quenching of the BL selectively excited at a fixed $\hbar \omega_{1}$ and found it to be activated with an energy of $22 \mathrm{meV}$. This activation energy is suggestive of involvement of shallow donors rather than deep donors in selectively excited BL. We note that an activation energy of about 10 times larger ( 0.3-0.4 eV) has been reported for the above-band-gap excited BL[7]. Such large difference in the thermal quenching activation energies between selectively-excited and above-band-gap excited PL was also found in the YL [5]. 


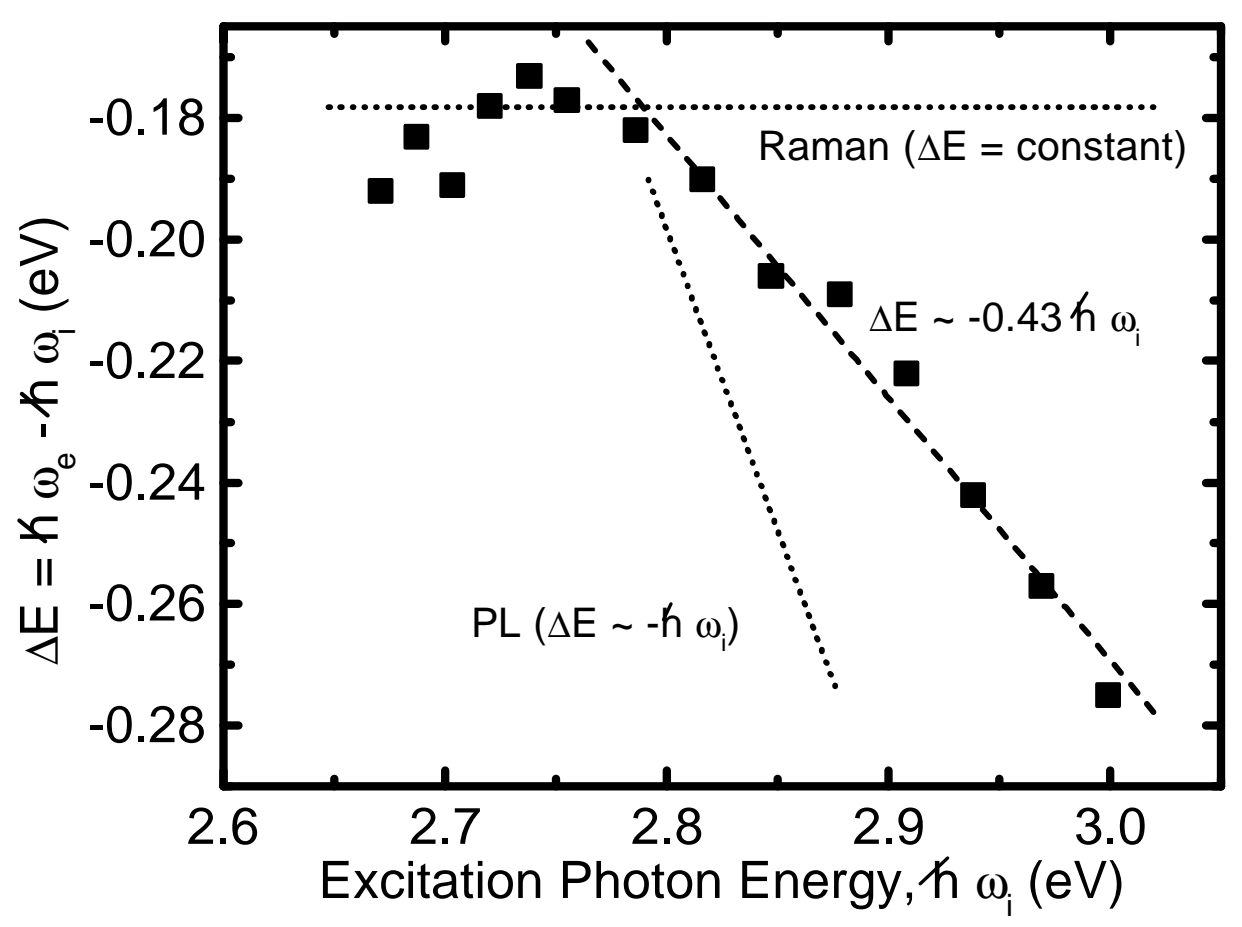

Fig 2. Summary of the selectively-excited blue luminescence peak positions (plotted as its shift from the excitation energy) in $\mathrm{GaN}: \mathrm{Mg}$ (at $\mathrm{T}=12 \mathrm{~K}$ ). The dashed line is a linear fit to data points for $\hbar \omega_{\mathrm{i}}>2.8 \mathrm{eV}$. The dotted lines represent the expected dependence for RS and PL peaks and have been labeled accordingly.

To understand our results we propose a "generalized" DAP recombination model which incorporates many of the features in previously proposed models. First we suggest that the exponential increase of the integrated PL intensity on $\hbar \omega_{1}$ reflects the existence of an Urbach tail in the absorption coefficient below the band gap. The cause of this band tailing in our GaN sample is clearly the fluctuation in band gap as a result of the heavy doping[8]. The idea that heavy self-compensation of the $\mathrm{Mg}$ acceptors in $\mathrm{GaN}$ may give rise to a strong fluctuating potential has been suggested[3,7]. The unusual dependence of the BL peak energy on $\hbar \omega_{1}$ depicted in Fig. 2 indicates that within the band tail states there is a "critical" energy (similar to a "mobility gap" in amorphous $\mathrm{Si}[8]$ ) occurring around $2.8 \mathrm{eV}$. For $\hbar \omega_{\mathrm{i}}<2.8 \mathrm{eV}$ the photo-excited carriers are strongly isolated or localized in distant centers with no energy relaxation between them. Since there is a high concentration of acceptors in our sample, we expect the electrons in the compensating donors to be isolated. Recombination of electrons from these isolated donors with the acceptor holes is "Franck-Condon shifted" from $\hbar \omega_{i}$ by a fixed amount of energy $\sim 180 \mathrm{meV}$ giving rise to the $\hbar \omega_{1}$ dependence in Fig. 2 which is similar to conventional RS. We note that $180 \mathrm{meV}$ is equal to the Franck-Condon shift deduced by Kaufmann et al. [9] from an analysis of the BL linewidth. It is approximately the energy of two LO phonons in GaN.

For $\hbar \omega_{1}>2.8 \mathrm{eV}$ we can explain the linear blue-shift of the BL emission peak with $\hbar \omega_{\mathrm{i}}$ in Fig. 2 in the same way as the linear blue-shift of the DAP peak energy with the log of the 
$11^{\text {th }}$ International Semiconducting and Insulating Materials Conference held at Canberra, Australia, 3-7 July, 2000.

excitation intensity which has been observed in highly nitrogen doped $\mathrm{ZnSe}$ [10]. In our case, the BL intensity and hence the photo-excited carrier concentration $(\mathrm{N})$ increases exponentially with $\hbar \omega_{\mathrm{i}}$. As a result $\log (\mathrm{N})$ increases linearly with $\hbar \omega_{\mathrm{i}}$ and so is the blue-shift of the BL peak energy. To understand the slope of $\sim 0.4$ in Fig. 2 requires a quantitative model for the dependence of the blue shift on $\log (\mathrm{N})$ such as the one recently proposed by Kuskovsky et al. [10]. Qualitatively the blue shift can be explained by an increase in the quasi-Fermi level of the donor electrons with $\mathrm{N}$. The change from a RS behavior to a PL behavior at $\hbar \omega_{1} \sim 2.8 \mathrm{eV}$ observed in Fig. 2 suggests that donors in heavily $\mathrm{Mg}$ doped $\mathrm{GaN}$ with energy below a certain "critical" energy are strongly localized and not in quasi-thermal equilibrium. On the other hand, electrons excited in donors above this energy can interact via hopping or tunneling and hence achieve a quasi-thermal equilibrium with well-defined quasi-Fermi levels. A more quantitative comparison between the model of Kuskovsky et al. [10] and our result will be presented elsewhere[11].

The different temperature dependence of selectively-excited BL and of the "normal" above-band-gap excited BL is not clear at this point and requires further investigation. One possible explanation is that in above band gap excitation the dominant non-radiative recombination centers are saturated. The larger activation energy of the above-band-gap excited BL represents the energy to thermally empty those traps.

In conclusion, we have found that selectively excited BL in heavily $\mathrm{Mg}$ doped $\mathrm{GaN}$ behaved differently than the BL excited by above band gap photons. The high $\mathrm{Mg}$ doping leaves to a fluctuating potential and an Urbach tail below the band gap. The BL behavior changes from Raman scattering to photoluminescence as a function of the excitation photon energy. These results suggest that donor electrons are isolated below a "critical" energy while those above this energy can achieve quasi-thermal equilibrium. The electrons in the isolated centers exhibit a Franck-Condon shift of $\sim 180 \mathrm{meV}$.

\section{Acknowledgments}

This work was supported by the Director, Office of Energy Research, Office of Basic Energy Sciences, Materials Sciences Division, of the US. Department of Energy under Contract No. DE-AC03-76SF00098.

\section{References}

[1] See for example, S. Nakamura and G. Fasal, The Blue Laser Diode (Springer-Verlag, Berlin, 1997).

[2] M. Godlewski et al., Physica B 273-274 (1999) 39.

[3] E. R. Glaser, et al. , Physica B 273-274 (1999) 58 and (unpublished).

[4] T. Suski, (unpublished).

[5] J. S. Colton et al., Appl. Phys. Lett. 75 (1999) 3273.

[6] E. Oh, H. Park and Y. Park, Appl. Phys. Lett. 72 (1998) 70.

[7] M.A. Reshchikov, G.-C. Yi, B.W. Wessels, Phys. Rev. B 59 (1999) 13176.

[8] See, for example, K. W. Böer, Survey of Semiconductor Physics (Van Nostrand Reinhold, New York, 1970).

[9] U. Kaufmann et al. Phys. Rev. B 59 (1999) 5561.

[10] J. Kuskovsky et al. Appl. Phys. Lett. 75 (1999) 1243 and also references therein.

[11] J. S. Colton and P.Y. Yu (unpublished). 